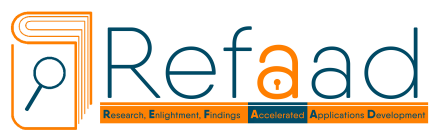

General Letters in Mathematics Vol. 4, No. 3, June 2018, pp.102-106

e-ISSN 2519-9277, p-ISSN 2519-9269

Available online at http:// www.refaad.com

https://doi.org/10.31559/glm2018.4.3.2

\title{
A Note on Some Unified Types of Open and Locally Closed Sets
}

\author{
Zanyar A. Ameen ${ }^{1}$ and Baravan A. Asaad ${ }^{2}$ \\ ${ }^{1}$ Department of Mathematics, College of Science, University of Duhok, IRAQ \\ ${ }^{2}$ Department of Computer Science, College of Science, Cihan University-Duhok, IRAQ \\ ${ }^{2}$ Department of Mathematics, Faculty of Science, University of Zakho, IRAQ \\ ${ }^{1}$ zanyar@uod.ac, ${ }^{2}$ baravan.asaad@uoz.edu.krd
}

Abstract. Recently, new types of (open) sets have been studied by some topologist. This note shows that these sets are identical to some other sets already exist in the literature.

Keywords: feebly open; b-open; $\alpha$-open; $\beta$-open; $b^{*}$-open; *b-open; $b^{* *-o p e n ; ~ * * b-o p e n ; ~ l o c a l l y ~ c l o s e d . ~}$ 2010 MSC No: 54A10.

\section{Introduction}

It is known that open sets are the fundamental building blocks of topology. They have experienced rapid development during the past sixty years, and nowadays it is a primary topic for many general topologists. The introduction of first type of open sets goes back to Levine [11] under the name of semiopen in 1963. Since then, $\beta$-open, preopen, $\alpha$-open, and b-open sets have been defined by Abd El-Monsef et al. [1, Mashhour et al. [13, Njastad [14, and Andrijevic [2], respectively. These types of open sets are the most well-known weak type of open sets. Then too many types of open sets (strong and weak) have been defined and studied. Some authors defined types of open sets without studying them well or comparing them to the closest types of open sets exist in the literature. In 1982 , Maheshwari 12 introduced the class of feebly open sets, and in 2012, Indira and Rekha [8] introduced *b-open and $*^{*}$ b-open sets. We prove that feebly open, ${ }^{*}$ b-open and ${ }^{* *}$ b-open sets are similar to the class of $\alpha$-open sets, see Theorem 2.6. In 2011, Bharathi et al. 3] defined the class of $b^{* *}$-open sets, and then, in 2016, Sayed and Mansour [16] introduced the class of $\mathrm{b}^{*}$-open sets. But it turns out that these two classes are the same as the class of $\beta$-open sets, see Theorem 2.3 .

The concept of locally closed sets is due to Kuratowski and Sierpinski [10, in 1921. Also, this type of sets has received a rapid growth. Many researchers used different types of open and closed sets to introduce new class of locally closed sets, including $([3,6]$, 7], 8], 9], [15]). We show that several classes of these sets are equal. For more details, see the results in Section III.

\section{Equivalence Types Of Open Sets}

Let $A$ be any subset of a topological space $(X, \tau), \operatorname{Int}(A)$ and $C l(A)$ denote the interior and the closure of $A$, respectively.

Definition 2.1. A subset $A$ of a topological space $(X, \tau)$ is said to be: 
1. $\beta$-open [1] if $A \subseteq C l(\operatorname{Int}(C l(A)))$.

2. preopen [13] if $A \subseteq \operatorname{Int}(C l(A))$.

3. semiopen [11] if $A \subseteq C l(\operatorname{Int}(A))$.

4. semiclosed [4] if $\operatorname{Int}(C l(A)) \subseteq A$.

5. $\alpha$-open [14] if $A \subseteq \operatorname{Int}(C l(\operatorname{Int}(A)))$.

6. b-open [2] if $A \subseteq C l(\operatorname{Int}(A)) \cup \operatorname{Int}(C l(A))$.

7. $b^{*}$-open [16] if $A \subseteq C l(\operatorname{Int}(C l(A))) \cup \operatorname{Int}(C l(A))$.

8. $b^{* *}$-open [3] if $A \subseteq \operatorname{Int}(C l(\operatorname{Int}(A))) \cup C l(\operatorname{Int}(C l(A)))$.

9. *b-open [8] if $A \subseteq C l(\operatorname{Int}(A)) \cap \operatorname{Int}(C l(A))$.

10. **b-open [8] if $A \subseteq \operatorname{Int}(C l(\operatorname{Int}(A))) \cap C l(\operatorname{Int}(C l(A)))$.

11. feebly open [12] if there exists an open set $U$ such that $U \subseteq A \subseteq s C l(U)$, where $s C l(A)$ is the intersection of all semiclosed sets containing $A$.

The complement of $\beta$-open (respectively, preopen, $\alpha$-open, b-open, $\mathrm{b}^{*}$-open, $\mathrm{b}^{* *}$-open, ${ }^{*} \mathrm{~b}$-open, ${ }^{* *} \mathrm{~b}$-open and feebly open) is said to be $\beta$-closed (respectively, preclosed, $\alpha$-closed, b-closed, $\mathrm{b}^{*}$-closed, $\mathrm{b}^{* *}$-closed, ${ }^{*} \mathrm{~b}$-closed, ${ }^{* *} \mathrm{~b}$ closed and feebly closed).

The results contained in this paper are based on the following lemma. We know that it is an elementary result but also important.

Lemma 2.2. [17, Ch.2] For any subsets $A, B$ of a topological space $(X, \tau)$, the following statements hold:

1. $\operatorname{Int}(A) \subseteq A$.

2. $A \subseteq C l(A)$.

3. $\operatorname{Int}(\operatorname{Int}(A))=\operatorname{Int}(A)$.

4. $C l(C l(A))=C l(A)$.

5. If $A \subseteq B$, then $\operatorname{Int}(A) \subseteq \operatorname{Int}(B)$.

6. If $A \subseteq B$, then $C l(A) \subseteq C l(B)$.

7. $\operatorname{Int}(C l(\operatorname{Int}(C l(A))))=\operatorname{Int}(C l(A))$.

8. $C l(\operatorname{Int}(C l(\operatorname{Int}(A)))) \subseteq C l(\operatorname{Int}(A))$.

9. $\operatorname{Int}(A) \subseteq \operatorname{Int}(C l(\operatorname{Int}(A))) \subseteq \operatorname{Int}(C l(A)) \subseteq \operatorname{Int}(C l(A)) \cup C l(\operatorname{Int}(A)) \subseteq C l(\operatorname{Int}(C l(A))) \subseteq C l(A)$.

10. $\operatorname{Int}(A) \subseteq \operatorname{Int}(C l(\operatorname{Int}(A))) \subseteq C l(\operatorname{Int}(A)) \subseteq \operatorname{Int}(C l(A)) \cup C l(\operatorname{Int}(A)) \subseteq C l(\operatorname{Int}(C l(A))) \subseteq C l(A)$.

From the above lemma, one can have the following relation between the most well-known weak types of open sets.

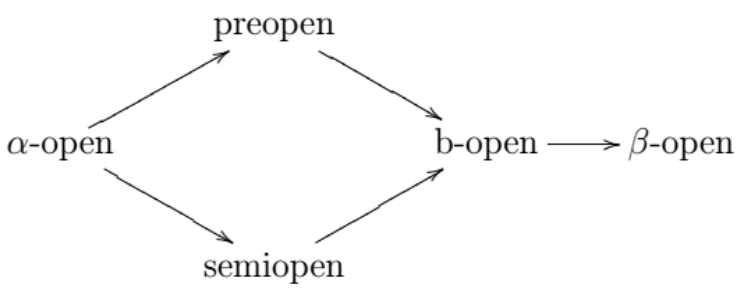

Diagram (A)

The above results tell us no matter how many times you take closure and interior of a set in the same manner as in Definition 2.1. or their unions or intersections, you will end up with one of the open sets in the diagram.

Now, it is time to give our main results. 
Theorem 2.3. For a subset $A$ of a topological space $(X, \tau)$, the following are equivalent:

1. A is $b^{* *}$-open;

2. $A$ is $b^{*}$-open; and

3. A is $\beta$-open.

Proof. (1) $\Longrightarrow(2)$ Let $A$ be $\mathrm{b}^{* *}$-open. Then $A \subseteq C l(\operatorname{Int}(C l(A))) \cup \operatorname{Int}(C l(\operatorname{Int}(A)))$. Since $\operatorname{Int}(C l(\operatorname{Int}(A))) \subseteq$ $\operatorname{Int}(C l(A))$ by Lemma $2.2(9)$, so $A \subseteq C l(\operatorname{Int}(C l(A))) \cup \operatorname{Int}(C l(A))$. Hence $A$ is b*-open.

$(2) \Longrightarrow(3)$ If $A$ is b*-open, then $A \subseteq C l(\operatorname{Int}(C l(A))) \cup \operatorname{Int}(C l(A))$. But $\operatorname{Int}(C l(A)) \subseteq C l(\operatorname{Int}(C l(A)))$, so $A \subseteq C l(\operatorname{Int}(C l(A)))$, which shows that $A$ is $\beta$-open.

$(3) \Longrightarrow(1)$ Let $A$ be a $\beta$-open set. So $A \subseteq C l(\operatorname{Int}(C l(A)))$. By Lemma 2.2 (10), Int $(C l(\operatorname{Int}(A))) \subseteq$ $C l(\operatorname{Int}(C l(A)))$, therefore $A \subseteq C l(\operatorname{Int}(C l(A))) \cup \operatorname{Int}(C l(\operatorname{Int}(A)))$. This proves that $A$ is $\mathrm{b}^{* *}$-open. We are done.

Lemma 2.4. A subset $A$ of a space $X$ is $\alpha$-open if and only if there is an open set $U$ such that $U \subseteq A \subseteq \operatorname{Int}(C l(U))$.

Proof. Suppose that $A$ is $\alpha$-open. So $A \subseteq \operatorname{Int}(C l(\operatorname{Int}(A)))$. Since $\operatorname{Int}(A) \subseteq A$ by Lemma $2.2(1)$, then $\operatorname{Int}(A) \subseteq$ $A \subseteq \operatorname{Int}(C l(\operatorname{Int}(A)))$. Set $U=\operatorname{Int}(A) \Longrightarrow U \subseteq A \subseteq \operatorname{Int}(C l(U))$ and $U$ is open.

Conversely; assume that there exists an open set $U$ such that $U \subseteq A \subseteq \operatorname{Int}(C l(U))$. We need to show that $A \subseteq \operatorname{Int}(C l(\operatorname{Int}(A)))$. Since $U$ is open and by assumption, we have $U \subseteq A$, so $U \subseteq \operatorname{Int}(A) \subseteq \operatorname{Int}(C l(U) \subseteq$ $\operatorname{Int}(C l(\operatorname{Int}(A)))$. But $A \subseteq \operatorname{Int}(C l(U))$. Therefore $A \subseteq \operatorname{Int}(C l(U)) \subseteq \operatorname{Int}(C l(\operatorname{Int}(A))$. Thus $A \subseteq \operatorname{Int}(C l(\operatorname{Int}(A)))$. This shows that $A$ is $\alpha$-open.

Lemma 2.5. [5, Lemma 2.1] If $A$ is an open subset of a space $X$, then $\operatorname{sCl}(A)=\operatorname{Int}(C l(A))$.

Theorem 2.6. For a subset $A$ of a topological space $(X, \tau)$, the following are equivalent:

1. A is **b-open;

2. $A$ is *b-open;

3. A is $\alpha$-open; and

4. A is feebly open.

Proof. (1) $\Longrightarrow(2)$ Let $A$ be ${ }^{* *}$ b-open. Then $A \subseteq \operatorname{Int}(C l(\operatorname{Int}(A))) \cap C l(\operatorname{Int}(C l(A)))$. Since $\operatorname{Int}(C l(\operatorname{Int}(A))) \subseteq$ $C l(\operatorname{Int}(C l(A)))$ by Lemma $2.2(9)$, so $A \subseteq \operatorname{Int}(C l(\operatorname{Int}(A)))$. By Lemma 2.2 (9) \& (10), Int $(C l(\operatorname{Int}(A))) \subseteq$ $\operatorname{Int}(C l(A))$ and $\operatorname{Int}(C l(\operatorname{Int}(A))) \subseteq C l(\operatorname{Int}(A))$, respectively. Therefore $A \subseteq \operatorname{Int}(C l(A)) \cap C l(\operatorname{Int}(A))$. Hence $A$ is $* \mathrm{~b}$-open.

$(2) \Longrightarrow(3)$ If $A$ is *b-open, then $A \subseteq C l(\operatorname{Int}(A)) \cap \operatorname{Int}(C l(A))$. Therefore $A \subseteq C l(\operatorname{Int}(A))$ and $A \subseteq \operatorname{Int}(C l(A))$. From the former, we have $C l(A) \subseteq C l(\operatorname{Int}(A))$ and then $\operatorname{Int}(C l(A)) \subseteq \operatorname{Int}(C l(\operatorname{Int}(A)))$. But $A \subseteq \operatorname{Int}(C l(A))$. So $A \subseteq \operatorname{Int}(C l(\operatorname{Int}(A)))$, which shows that $A$ is $\alpha$-open.

$(3) \Longrightarrow(4)$ Follows from Lemmas 2.4 and 2.5

$(4) \Longrightarrow(1)$ By (4) there exists an open set $U$ such that $U \subseteq A \subseteq s C l(A)$. Since $U \subseteq A$ and $U$ is open, so $U \subseteq \operatorname{Int}(A) \Longrightarrow C l(U) \subseteq C l(\operatorname{Int}(A)) \Longrightarrow \operatorname{Int}(C l(U)) \subseteq \operatorname{Int}(C l(\operatorname{Int}(A)))$. Therefore, by assumption and Lemma 2.5. $A \subseteq s C l(U)=\operatorname{Int}(C l(U)) \subseteq \operatorname{Int}(C l(\operatorname{Int}(A)))$. But, by Lemma 2.2 $(10), \operatorname{Int}(C l(\operatorname{Int}(A))) \subseteq C l(\operatorname{Int}(C l(A)))$, so $\operatorname{Int}(C l(\operatorname{Int}(A)))=\operatorname{Int}(C l(\operatorname{Int}(A))) \cap C l(\operatorname{Int}(C l(A)))$. Hence $A \subseteq \operatorname{Int}(C l(\operatorname{Int}(A))) \cap C l(\operatorname{Int}(C l(A)))$. This proves that $A$ is **b-open.

\section{Equivalence Types Of Localized Closed Sets}

In this section, we define some concepts without studying as we were not able to find them in the literature during preparing the present paper. Probably some authors defined them under odd names. We follow topologists for naming them to be standard.

Definition 3.1. A subset $A$ of a topological space $(X, \tau)$ is called:

1. locally $b^{* *}$-closed [3] if $A=U \cap F$, where $U$ is open and $F$ is $b^{* *}$-closed.

2. locally $b^{*}$-closed if $A=U \cap F$, where $U$ is open and $F$ is $b^{*}$-closed. 
3. locally ${ }^{*} b$-closed [8, 9], if $A=U \cap F$, where $U$ is open and $F$ is ${ }^{*} b$-closed.

4. locally ${ }^{* *} b$-closed [8] if $A=U \cap F$, where $U$ is open and $F$ is ${ }^{* *} b$-closed.

5. locally $\beta$-closed if $A=U \cap F$, where $U$ is open and $F$ is $\beta$-closed.

6. locally $\alpha$-closed if $A=U \cap F$, where $U$ is open and $F$ is $\alpha$-closed.

Theorem 3.2. For a subset $A$ of a topological space $(X, \tau)$, the following are equivalent:

1. A is locally $b^{* *}$-closed;

2. A is locally $b^{*}$-closed; and

3. A is locally $\beta$-closed.

Proof. Follows from Theorem 2.3

Theorem 3.3. For a subset $A$ of a topological space $(X, \tau)$, the following are equivalent:

1. A is locally **b-closed;

2. A is locally *b-closed; and

3. A is locally $\alpha$-closed.

Proof. Follows from Theorem 2.6

If we are allowed to introduce the following types of locally closed sets, then we would have the results below:

Definition 3.4. A subset $A$ of a topological space $(X, \tau)$ is called:

1. $b^{* *}$-locally closed if $A=U \cap F$, where $U$ is $b^{* *}$-open and $F$ is $b^{* *}$-closed.

2. $b^{*}$-locally closed if $A=U \cap F$, where $U$ is $b^{*}$-open and $F$ is $b^{*}$-closed.

3. *b-locally closed if $A=U \cap F$, where $U$ is *b-open and $F$ is *b-closed.

4. **b-locally closed if $A=U \cap F$, where $U$ is **b-open and $F$ is ${ }^{*} b$-closed.

5. $\beta$-locally closed [6] if $A=U \cap F$, where $U$ is $\beta$-open and $F$ is $\beta$-closed.

6. $\alpha$-locally closed [7] if $A=U \cap F$, where $U$ is $\alpha$-open and $F$ is $\alpha$-closed.

7. feebly locally closed [15] if $A=U \cap F$, where $U$ is feebly open and $F$ is feebly closed.

Theorem 3.5. For a subset $A$ of a topological space $(X, \tau)$, the following are equivalent:

1. A is $b^{* *}$-locally closed;

2. A is $b^{*}$-locally closed; and

3. A is $\beta$-locally closed.

Proof. Follows from Theorem 2.3

Theorem 3.6. For a subset $A$ of a topological space $(X, \tau)$, the following are equivalent:

1. A is **b-locally closed;

2. A is *b-locally closed; and

3. A is $\alpha$-locally closed.

4. A is feebly locally closed.

Proof. Follows from Theorem 2.6.

Remark 3.7. Notice that the results presented in this note can be generalized to the notions of fuzzy, nano, ideal, soft topology etc.

ACKNOWLEDGEMENTS. We wish to thank the referees for their valuable comments and suggestions. 


\section{References}

[1] M. E. Abd El-Monsef, S. N. El-Deeb and R. A. Mahmoud, $\beta$-open sets and $\beta$-continuous mappings, Bull. Fac. Sci. Assuit. Univ., 12 (1) (1983), 1-18.

[2] D. Andrijevic, On b-open sets, Matematicki Vesnik, 48(1-2) (1996), 59-64.

[3] S. Bharathi, K. Bhuvaneshwari, N. Chandramathi, On locally b**-closed sets, International Journal of Mathematical Sciences and Applications, 1(2) (2011), 636-641.

[4] S. G. Crossley, S. K. Hildebrand. Semi-closure. Texas J. Sci., (22) (1971), 99-112.

[5] G. Di Maio and T. Noiri, On s-closed spaces,Indian J. Pure Appl. Math., 18 (3) (1987), 226-233.

[6] Y. Gnananmbal and K. Balachandran, $\beta$-locally closed sets and $\beta$-LC-continuous functions, Mem. Fac. Sci. Kochi Univ. Ser. A, Math., 19 (1998), 35-44.

[7] Y. Gnanambal, K. Balachandran and R. Devi, $\alpha$-locally closed sets and $\alpha$-LC-continuous functions, preprint.

[8] T. Indira and K. Rekha, On locally ${ }^{* *}$ b-closed sets, Proceedings of the Heber International Conference on Applications of Mathematics and Statistics (HICAMS) (2012).

[9] T. Indira and K. Rekha, Decomposition of continuity via *b-open set, Communicated (2012).

[10] C. Kuratowski and W. Sierpinski, Sur les differences de deux ensembles fermes, Tohoku Math. J., 20 (1921), $22-25$.

[11] N. Levine, Semi-open sets and semi-continuity in topological spaces, Amer. Math. Monthly, 70 (1) (1963), 36-41. https://doi.org/10.2307/2312781

[12] S. N. Maheshwari and P. C. Jain, Some new mappings, Mathematica, 24 (47) (1982), 53-55.

[13] A. S. Mashhour, M. E. Abd El-Monsef and S. N. El-Deeb, On precontinuous and week precontinuous mappings, Proc. Math. Phys. Soc. Egypt, 53 (1982), 47-53.

[14] O.Njastad, On some classes of nearly open sets, Pacific J. Math., 15 (3) (1965), 961-970. https://doi.org/10.2140/pjm.1965.15.961

[15] A. A. Nasef, Feebly locally closed sets and feebly LC-continuous functions, to appear.

[16] M E L Sayed and F H A L Mansour, New Near Open Set in Topological Space, Journal of Physical Mathematics, 7 (4) (2016). https://doi.org/10.4172/2090-0902.1000204

[17] S. Willard, General topology, Courier Corporation, 2004. 